\title{
Cs-corrected STEM Study of Structure and Chemistry of Au/Ni/GaAs, $\mathrm{Au} / \mathrm{Cu} / \mathrm{CdTe}$ and Pt/YSZ Interfaces
}

\author{
K. Sun \\ Department of Materials Science and Engineering, University of Michigan, Ann Arbor, MI48109
}

Interface structure and chemistry play very important roles in material's performance especially for thin film materials. Bcc-Ni thin film has been successfully grown on GaAs substrate that exhibits ferromagnetic property different from its fcc-Ni counterpart [1]. As back contact materials $\mathrm{Cu}$ and $\mathrm{Au}$ have been normally deposited on $\mathrm{CdTe} / \mathrm{CdS}$ solar cells [2]. However, the roles of $\mathrm{Cu}$ in improving contact property and in cell degradation are still not clear. Pt/YSZ has been widely used as electrode in solid oxide fuel cells and its performance is believed to be strongly dependent on the triple phase boundaries where catalytic reaction occurs [3]. To understand the properties of the above mentioned materials systems, advanced techniques are needed to study the structures and chemistries of the interfaces in the materials.

A $5 \mathrm{~nm}$ thick single crystal bcc-Ni film capped with a $2 \mathrm{~nm}$ thick Au film was grown on GaAs substrate by MBE. Typical magnetron sputtering method was used for depositing $\mathrm{Cu}$ and $\mathrm{Au}$ on the surface of $\mathrm{CdTe} / \mathrm{CdS}$ films on top of $\mathrm{TCO} /$ glass substrate. The cells were then thermally treated at $200^{\circ} \mathrm{C}$ in the dark under normal air or dry $\mathrm{N}_{2}$ atmospheres. The thicknesses of the $\mathrm{Cu}$ and $\mathrm{Au}$ layers are $3.5 \mathrm{~nm}$ and $20 \mathrm{~nm}$, respectively. Thin Pt film with thickness of $1.2 \mathrm{~nm}$ was grown on YSZ substrate at $600{ }^{\circ} \mathrm{C}$ by sputter deposition. A JEOL $2100 \mathrm{FS}$ with a probe corrector was used for the study of the materials. The lens conditions were set to define a probe size of 0.1 $\mathrm{nm}$ for both high-angle annual dark-field (HAADF) imaging and electron energy loss spectroscopy (EELS) and EDS analysis.

Figure 1 displays some preliminary results obtained from the $\mathrm{Au} / \mathrm{Ni} / \mathrm{GaAs}$ sample. The HAADF image in Fig. 1A shows that a single crystal Ni layer has been grown epitaxially on GaAs. Fourier filtered HAADF image of the outlined region in Fig. 1A is shown in Fig.1B which shows much clearly the epitaxial growth of the Ni layer on GaAs. EDS and EELS profile analyses of the thin film material were also performed which indicates no inter-diffusion has occurred in the asgrown thin film material. However, after a beam shower was made for 30 minutes to try to prevent the analyzed area from contamination, the diffusion of Ni into the GaAs substrate had occurred during our study. The results are shown in Fig. 2 which clearly demonstrates the electron beam irradiation induced diffusion of Ni into the semiconductor substrate. EDS profile shows that no Au diffusion has occurred. It should be indicated that the beam shower method has been routinely used which has been thought to be an efficient way to keep TEM specimen clean in TEM. The present results clearly show that electron beam irradiation induced material's structure change is not unique for metallic clusters [4] especially when an aberration-free TEM is used. Detailed study on the interface structure and chemistry of the materials as well as the beam effect on the materials are ongoing using our newly installed Cs-corrected microscope.

\section{References}

[1] C.S. Tian, et al., Phys. Rev. Lett. 94 (2005) 137210.

[2] X.X. Liu et al., The $34^{\text {th }}$ IEEE Photovoltaic Specialists Conference (PVSC), June 7-12 (2009) Philadelphia, PA.

[3] N. Nakagawa et al., Solid State Ionics 40-41 (1990) 411.

[4] P.E. Batson, Microsc. Microanal. 14 (2008) 89. 
[5] This research was supported by the DoE and Ford Motor Company. Prof. X.F. Jin from Fudan University, China, Prof. A.D. Compaan from University of Toledo and Dr. J. Yang from Ford Motor Company supplied the materials. The aids of Dr. D. Blom from University of South Carolina and Dr. M. Kawasaki from JEOL USA are gratefully acknowledged.
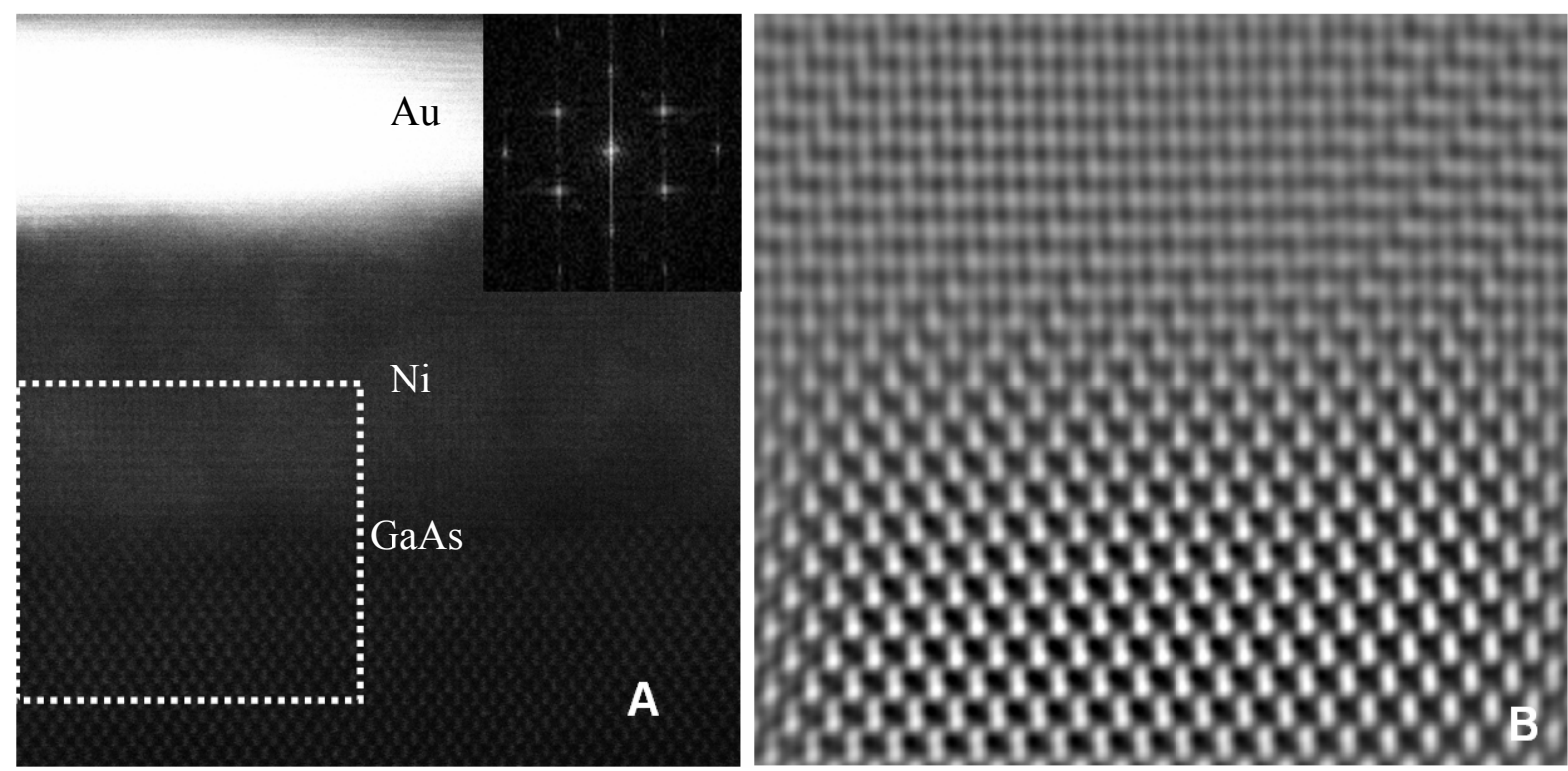

FIG. 1. (A) HAADF image showing the growth of single crystal bcc-Ni layers on GaAs. The inset is a FFT of the outlined area. Enlarged Fourier filtered image of the outlined region is shown in (B).
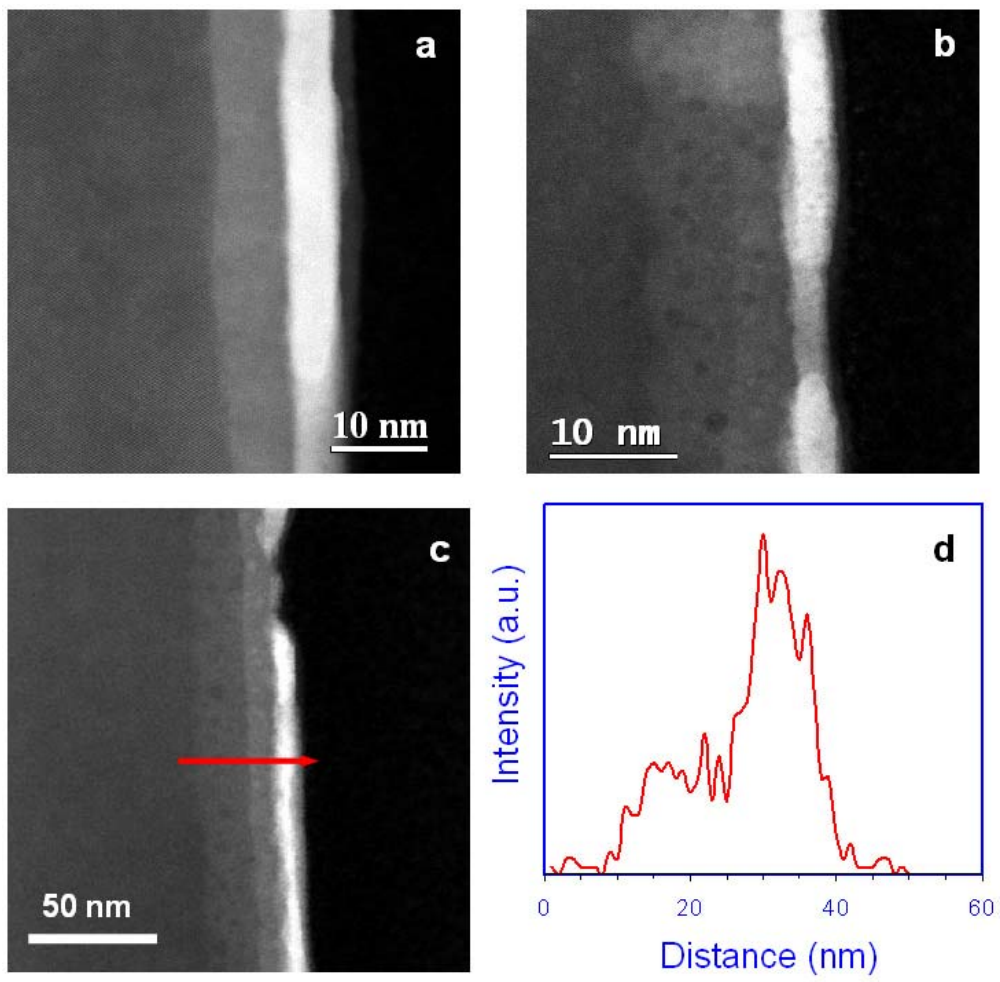

FIG. 2. (a) and (b) are HAADF images taken before and after the 30 minute beam shower has been made; (c) is also a HAADF image taken from another area of the specimen having experienced the beam shower. Ni EELS profile (shown in d) was performed along the red arrow marked in (c). 